\title{
Consumo intensivo de alcohol en adolescentes: prevalencia, conductas de riesgo y variables asociadas
}

\section{Binge Drinking among Adolescents: Prevalence, Risk Practices and Related Variables}

\author{
Sandra Golpe*, Manuel Isorna*, Carmen Barreiro*, Teresa Braña*, Antonio Rial*. \\ *Universidad de Santiago de Compostela, Santiago de Compostela, España.
}

\section{Resumen}

Según la última Encuesta sobre uso de drogas en Enseñanzas Secundarias (ESTUDES 2014-2015), los niveles de consumo tanto de alcohol como de otras sustancias han disminuido en España en los últimos años. No obstante, siguen siendo preocupantes los datos referidos al consumo intensivo de alcohol (CIA), sobre todo habida cuenta las graves repercusiones asociadas a este patrón. El objetivo del presente trabajo ha sido analizar el consumo intensivo de alcohol entre los adolescentes, ofreciendo datos actualizados no sólo de su prevalencia, sino también de sus consecuencias y posibles factores de pronóstico. Para ello se utilizó una metodología correlacional, consistente en la realización de una encuesta a estudiantes de ESO, Bachillerato y FP de grado medio. La muestra final estuvo compuesta por 3.419 adolescentes gallegos de entre 12 y 18 años $(M$ $=14,57 ; S D=1,76)$. Los resultados obtenidos revelan que el CIA es una práctica frecuente y globalizada, con escasas diferencias a nivel sociodemográfico, pero asociada a un amplio abanico de conductas de riesgo. Por otra parte, variables como las expectativas de consumo, el consumo entre los pares y en el entorno familiar, así como la hora de llegada a casa o el dinero disponible han sido identificadas como interesantes factores de pronóstico que debieran ser tenidos en cuenta en el plano preventivo.

Palabras clave: Adolescentes; Alcohol; Consumo intensivo; AUDIT.

\begin{abstract}
According to the last Survey on Drug Use among Secondary School Students (ESTUDES 2014-2015), consumption levels of alcohol and other substances have decreased in the last years in Spain. However, available data on binge drinking remain worrying, given the negative consequences related with this pattern. The aim of this paper is to analyse binge drinking among adolescents, providing updated data on prevalence in addition to information about the consequences and some predictive factors of binge drinking. A correlational method was used for this purpose, comprised of administering a survey to Compulsory Secondary School, High School and Vocational Training students. Based on a sample of 3,419 Galician adolescents aged between 12 and 18 years $(M=14.57 ; S D=1.76)$, the results show that binge drinking is a common and global practice, with few socio-demographic differences but related with a wide range of risk practices. Furthermore, variables such as consumption expectancies, consumption by family and friends, as well as curfew time and allowance money have been identified as interesting predictive factors that should be taken into account at the preventive level.

Keywords: Adolescents; Alcohol; Underage drinking; Binge drinking; AUDIT.
\end{abstract}


$\mathrm{E}$ 1 consumo abusivo de alcohol entre los jóvenes constituye uno de los principales problemas de salud pública en España, tal y como se recogía en la Estrategia Nacional sobre Drogas 2009-2016 (Plan Nacional sobre Drogas, 2009a). Lo mismo puede decirse a nivel europeo con la estrategia puesta en marcha por el Consejo de Europa en materia de lucha contra la droga 2013-2020 (Consejo de la Unión Europea, 2013). Si bien los niveles de consumo tanto de alcohol como de otras drogas han descendido en los últimos años, las cifras de prevalencia siguen siendo elevadas, especialmente en el caso del alcohol, que sigue erigiéndose como la sustancia psicoactiva más consumida. Según datos del European School Project on Alcohol and Other Drugs (ESPAD 2011) (Hibell et al., 2012) un $79 \%$ de los estudiantes de entre $15 \mathrm{y}$ 16 años habían consumido alcohol en los últimos 12 meses y un $57 \%$ lo había hecho en el último mes. En España los resultados de la última Encuesta Estatal sobre uso de Drogas en Enseñanzas Secundarias (ESTUDES 2014-2015) (Plan Nacional sobre Drogas, 2016) señalan que el 76,8\% de los adolescentes entre 14 y 18 años consumieron alcohol en el último año y un $68,2 \%$ en el último mes.

En este contexto, una de las cuestiones que más sigue preocupando a profesionales e investigadores es la instauración de un patrón de consumo caracterizado por la ingesta de grandes cantidades de alcohol en cortos espacios de tiempo, principalmente en fin de semana y que suele llevar a la embriaguez (Anderson, 2007; Calafat, 2007; Cortés, Espejo, y Giménez, 2007). La literatura anglosajona denomina generalmente a este patrón de consumo como binge drinking $(B D)$ aunque en España se ha optado por referirse al Consumo Intensivo de Alcohol (CIA) (Rodríguez-Martos y Rosón, 2008). Según la Organización Mundial de la Salud (OMS, 2004) el CIA se definiría como la ingesta, por parte de un adulto, de al menos 60 gramos de alcohol (6 Unidades de Bebida Estándar -UBE- en España) en una ocasión de consumo. No obstante, son muchas las dificultades que se plantean a la hora de hacer operativo el CIA a partir de esta definición. En primer lugar, la falta de consenso en lo que puede considerarse una Unidad de Bebida Estándar (UBE) hace que el criterio referido al número de consumiciones alcohólicas por ocasión de consumo varíe de un país a otro (Mongan y Long, 2015; Parada et al., 2011). Asimismo, la no especificación del período de tiempo que se considera "una única ocasión" hace que diversos autores hayan planteado la necesidad de tener en cuenta los niveles de concentración de alcohol en sangre, lo que supone incorporar la duración en la definición del CIA (National Institute on Alcohol Abuse and Alcoholism [NIAAA], 2004). A la luz de estas consideraciones, autores como Parada et al. (2011), proponen considerar el CIA como el consumo de 6 o más bebidas alcohólicas para hombres ( 5 o más para mujeres) en una única ocasión de consumo (en un período de 2 horas) al menos una vez en los últimos 30 días. No obstante, cuando se trabaja con adolescentes se ha señalado la conveniencia de rebajar a 3 el número de bebidas alcohólicas por ocasión de consumo, puesto que los niveles de concentración de alcohol en sangre que estos alcanzan con el mismo número de consumiciones es mucho mayor que el de los adultos (Donovan, 2009).

Esta falta de consenso a la hora de hacer operativo el CIA y el empleo de muestras muy heterogéneas han provocado que los diversos estudios epidemiológicos hayan ofrecido cifras de prevalencia en ocasiones muy dispares. Así por ejemplo, datos recientes procedentes de la National Survey on Drug Use and Health (Hedden et al., 2015) señalan que el 6,1\% de los adolescentes entre 12 y 17 años han realizado un consumo intensivo de alcohol en el último mes en Estados Unidos. Por su parte, los resultados del ESPAD 2011 indican que el $39 \%$ de los estudiantes europeos entre 15 y 16 años han consumido alcohol de manera intensiva también en el último mes, mientras que en España un $32,2 \%$ de los estudiantes entre 14 y 18 años practicaron binge drinking en los últimos 30 días y un 22,2\% se emborracharon (Plan Nacional sobre Drogas, 2016).

En cualquier caso, lo que parece claro es que el consumo intensivo de alcohol constituye un grave problema sociosanitario con claras consecuencias negativas. Así, por ejemplo, algunos trabajos han constatado una estrecha relación entre el CIA y daños orgánicos a largo plazo, tales como cirrosis, hipertensión y enfermedades coronarias (Anderson, Cremona, Paton, Turner, y Wallace, 1993; Marmot, 2001; Pincock, 2003). No menos importantes son las alteraciones que se producen a nivel cerebral, tanto desde el punto de vista estructural como funcional (Cadaveira, 2009; López-Caneda et al., 2014; Tapert, 2007), siendo numerosos los trabajos que han documentado las posibles repercusiones neurocognitivas y neuroconductuales asociadas a este patrón de consumo (Cadaveira, 2010; Guerri, 2010; Guerri y Pascual, 2010; Tapert y Brown, 1999; Ziegler et al., 2005). La literatura también señala que los jóvenes que realizan un consumo intensivo de alcohol tienen mayor probabilidad de verse implicados en numerosos comportamientos de riesgo como peleas (Swahn, Simon, Hamming, y Guerrero, 2004; Wechsler, Davenport, Dowdall, Moeykens, y Castillo, 1994), conducir bajo los efectos del alcohol (Adams, Evans, Shreffler, y Beam, 2006; Windle, 2003), tener problemas con la policía, ser víctima de atracos o robos, participar en prácticas sexuales de riesgo (DeCamp, Gealt, Martin, O’Connell, y Visher, 2015; Huang, Jacobs, y Deverensky, 2010) o presentar un peor rendimiento académico (Miller, Naimi, Brewer, y Jones, 2007). Asimismo, trabajos como el de Jones, Oeltmann, Wilson, Brener, y Hill (2001) o el de Miller et al. (2007), han encontrado una estrecha relación entre el consumo intensivo de alcohol y el consumo de otras sustancias, e incluso se ha sugerido que el CIA durante la adolescencia es un factor de riesgo para el desarrollo posterior de abu- 
so/dependencia de alcohol en la edad adulta (Chambers, Taylor y Potenza, 2003; García-Moreno, Expósito, Sanhueza, y Angulo, 2008; Petit, Maurage, Kornreich, Verbanck, y Campanella, 2014). Una variable que incide en la probabilidad de aparición de muchas de estas consecuencias y en su gravedad es la edad a la que los adolescentes se inician en el consumo de alcohol (Motos, Cortés, Giménez, y Cadaveira, 2015). Tanto es así que retrasar la edad de inicio aparece contemplado como uno de los objetivos del Plan de Acción sobre Drogas 2013-16 (Plan Nacional sobre Drogas, 2009b) (objetivo general 4), así como en diferentes planes autonómicos, como sucede en el Plan de Trastorno Adictivos de Galicia 2011-2016 (Xunta de Galicia, 2010) (objetivo 1.3). Pese a ello, el principal sistema de información del que disponemos a nivel estatal respecto a esta práctica, el ESTUDES, utiliza un marco muestral limitado que incluye a adolescentes de entre 14 y 18 años. Parecería, por tanto, de interés poder disponer de datos empíricos referidos a la prevalencia del CIA entre los más jóvenes (12-13 años), sobre todo habida cuenta de que la edad de inicio en el consumo de alcohol se situaba ya en el año 2014 en los 13,9 años (Plan Nacional sobre Drogas, 2014).

Las importantes implicaciones que el CIA puede acarrear tanto a nivel clínico como psicosocial justifica además que investigadores, profesionales e instituciones centren buena parte de sus esfuerzos en desarrollar medidas preventivas que disminuyan los niveles de consumo de alcohol y, en particular, de esta nueva modalidad de consumo en forma de atracón. Para ello resulta clave ser capaces de identificar las posibles variables asociadas. Respecto al posible papel de las variables sociodemográficas distintos trabajos coinciden en señalar que los chicos tienden a consumir de manera más intensiva que las chicas (Fuller-Thomson, Sheridan, Sorichetti, y Mehta, 2013; Peralta, Steele, Nofziger, y Rickles, 2010) y que la prevalencia del CIA se incrementa con la edad, alcanzando sus niveles más altos en la adultez temprana (alrededor de los 20 años) (Mota et al., 2010; Windle, Mun, y Windle, 2005). Más allá del género o de la edad, el CIA se ha asociado también con variables personales, tales como un estilo de afrontamiento evitativo (Doumas, Turrisi, y Wright, 2006; Pirkle y Richter, 2006), una baja percepción del riesgo (Parada et al., 2011) o unas expectativas positivas respecto a los efectos del consumo de alcohol (Durkin, Wolfe, y Clark, 2005; McBride, Barret, Moore, y Schonfeld, 2014). También son muchos los trabajos que relacionan el CIA con el consumo de alcohol por parte de los iguales (Coleman y Cater, 2005; Stickley et al., 2013) y con variables del entorno familiar, como pueden ser una actitud parental favorable al consumo de alcohol (Jander, Mercken, Crutzen, y De Vries, 2013), el hecho de pertenecer a una familia desestructurada (Fuller-Thomson et al., 2013) o altos niveles de consumo por parte de los padres (Espada, Pereira, y García-Fernández, 2008; Pons y Berjano, 1999). Asimismo, existen otras variables como el dinero disponible, el estatus socioeconómico o la hora de llegada a casa que, si bien cuentan con menor evidencia empírica en el contexto del CIA, también han sido relacionadas con el consumo de alcohol entre los jóvenes (Humensky, 2010; Plan Nacional sobre Drogas, 2014; Varela, Marsillas, Isorna, y Rial, 2013).

Habida cuenta del interés que el tema sigue suscitando a diferentes niveles, el presente trabajo se ha planteado con el objetivo analizar el consumo intensivo de alcohol entre la población adolescente de la comunidad gallega, siendo éste definido en base a tres criterios: a) el consumo de 6 o más bebidas alcohólicas por ocasión de consumo, en un intervalo de 2 horas (que refleja criterio defendido por Parada et al., 2011); b) el consumo de 3 o más bebidas alcohólicas por ocasión de consumo (2 horas), que recoge la postura de aquellos que señalan la conveniencia de disminuir el número de bebidas alcohólicas cuando se trata de adolescentes; y c) el haberse emborrachado, con el que se pretende evaluar el componente más subjetivo del consumo. En definitiva, en primer lugar, se pretende disponer de nuevos datos acerca de la prevalencia de esta práctica entre los adolescentes, ampliando el marco muestral desde los 12 a los 18 años, a la vez que se analizan dichas prevalencias por segmentos poblacionales en función del género, la edad, la titularidad de centro, el entorno de residencia o el nivel de estudios de los padres. En segundo lugar, se intentan aportar nuevas evidencias respecto a las consecuencias o riesgos de un consumo intensivo de alcohol, que sirvan para reforzar la trascendencia de este fenómeno. Por último, se intentan identificar posibles factores de pronóstico, con la finalidad de orientar el trabajo a nivel preventivo.

\section{Método}

\section{Participantes}

Para dar cuenta del objetivo señalado se recurrió a una metodología correlacional. Concretamente se llevó a cabo una encuesta entre la población de estudiantes de Educación Secundaria Obligatoria (ESO), Bachillerato y Ciclos Formativos de grado medio de la comunidad autónoma de Galicia (aproximadamente 140.000 individuos). Para la selección de la muestra se utilizó un muestreo bietápico: por conglomerados, para la selección de las unidades de primer nivel (centros educativos) y por cuotas, según género y ciclo, para la selección de las unidades de segundo nivel (individuos). Un total de 37 centros de titularidad tanto pública como privada/concertada y de las cuatro provincias gallegas fueron seleccionados aleatoriamente, respetando las cuotas existentes a nivel poblacional. La muestra final estuvo compuesta por un total de 3.419 adolescentes $(50,6 \%$ hombres y $49,4 \%$ mujeres) de edades comprendidas entre los 12 y 18 años $(M=14,57$ y $S D=1,76)$. De estos, 2236 asistían a colegios públicos y 1183 a colegios privados o concertados. El $73,3 \%$ se encontraban cursando la ESO $(38,2 \%$ en el primer 
ciclo y $35,1 \%$ en el segundo), el 20,4\% Bachillerato y el 6,2\% FP Básica (PCPI) o un Ciclo Formativo de grado medio.

\section{Instrumento}

Los datos fueron recogidos mediante un cuestionario elaborado expresamente para el presente estudio en el que se incluían preguntas agrupadas en cinco bloques: (1) un primer bloque extraído de la Encuesta Estatal sobre Uso de Drogas en Estudiantes de Enseñanzas Secundarias (ESTUDES 2010) (Plan Nacional sobre Drogas, 2011) referido a los hábitos de consumo tanto de alcohol como de otras sustancias (en el último año y en el último mes); (2) un segundo bloque que incluía preguntas relativas al consumo intensivo de alcohol. La controversia existente respecto a la operativización de este constructo nos llevó a optar por el empleo de tres indicadores diferentes, dos de ellos cuantitativos (a) haber consumido 3 o más bebidas alcohólicas en una misma ocasión (en un intervalo de 2 horas) y (b) haber consumido 6 o más bebidas alcohólicas en una misma ocasión (2 horas) - y otro de carácter subjetivo - (c) haberse emborrachado; (3) un tercer bloque que incluía el Alcohol Use Disorder Identification Test (AUDIT) en su versión autoadministrada (Rial et al., 2015) para estimar el consumo de riesgo de alcohol y cuya consistencia interna en el presente trabajo resultó satisfactoria $(\alpha=, 77)$; (4) un cuarto bloque extraído del European School Survey Project on Alcohol and Other Drugs (ESPAD 2011) (Hibell et al., 2012) referido a posibles problemas asociados al consumo de alcohol; (5) un quinto bloque de preguntas de elaboración propia (si bien tomando como base los cuestionarios utilizados en el ESTUDES y el ESPAD), referidas a variables asociadas al CIA que la literatura ha puesto de relieve, tales como la hora de llegada a casa, el dinero disponible, las creencias y expectativas, el consumo en el entorno familiar o entre los iguales; y, por último, información referida a variables sociodemográficas como el género, la edad, la titularidad del centro, el entorno de residencia o el nivel de estudios de los padres.

\section{Procedimiento}

Los datos fueron recogidos en las propias aulas de los centros, en grupos reducidos (entre 15 y 20 individuos), mediante un cuestionario que cada estudiante debía cumplimentar de manera individual. La recogida de la información fue realizada por un equipo de psicólogas con experiencia acreditada en la realización de este tipo de tareas. Cada sujeto fue informado de la finalidad del estudio, así como la confidencialidad y anonimato de sus respuestas. Se contó con el consentimiento y la colaboración tanto de la dirección de los centros, como de las respectivas asociaciones de madres y padres de alumnos. La participación fue totalmente voluntaria y el tiempo de cumplimentación del cuestionario fue de aproximadamente 20 minutos. El trabajo contó además con la aprobación del Comité de Bioética de la Universidad de Santiago de Compostela.

\section{Análisis de datos}

En total fueron recogidos 3714 cuestionarios. Fruto de la depuración de la base de datos inicial fueron eliminados 295 casos, bien por presentar un volumen excesivo de valores ausentes (15), un patrón incoherente de respuesta (22) o por encontrarse fuera del rango de edad establecido (12-18 años) (258). Las diferencias entre consumidores intensivos y no intensivos fueron analizadas mediante una tabulación bivariada, con la aplicación de los contrastes oportunos en función de la naturaleza de las variables: pruebas $t$ de Student para la comparación de medias y coeficientes eta cuadrado $\left(\eta^{2}\right)$ para calcular el tamaño del efecto en variables cuantitativas, así como contrastes $\chi^{2}$ para la comparación de porcentajes y coeficientes phi $(\phi)$ y de contingencia (CC) para calcular el tamaño del efecto en variables cualitativas. Los análisis fueron realizados con el paquete estadístico IBM SPSS Statistics 20.

\section{Resultados}

Un primer dato de interés que se recoge en la Tabla 1 es que 6 de cada 10 adolescentes entre 12 y 18 años han bebido alcohol en el último año $(58,7 \%)$. Respecto al consumo intensivo de alcohol cabe señalar que el $24,5 \%$ ha consumido 6 o más bebidas alcohólicas en una única ocasión en el último año, cifra que desciende al 12,8\% si nos referimos al último mes. No obstante, si evaluamos el consumo intensivo a partir de la ingesta de 3 o más bebidas alcohólicas en una única ocasión, el porcentaje de consumidores en el último año y en el último mes se duplica $(41,8 \%$ y $25 \%$, respectivamente). Asimismo, hay un $34,4 \%$ de los adolescentes que afirman haberse emborrachado en el último año y un 16,5\% dice haberlo hecho en los últimos 30 días. Para favorecer la comparabilidad de los datos obtenidos en el presente trabajo con los proporcionados por el ESTUDES 2014-2015 (en el que se entrevistan únicamente a estudiantes de entre 14 y 18 años), en esta misma Tabla 1 se presentan los datos específicos para dicho grupo de edad. Como se puede comprobar, los porcentajes son considerablemente mayores que en caso de la muestra global (que incluye también la franja de 12-13 años), pero similares a los del ESTUDES, donde el porcentaje de sujetos que se

Tabla 1. Prevalencia del CIA en Galicia.

\begin{tabular}{lcccc}
\hline & \multicolumn{2}{c}{$\mathbf{1 2 - 1 8}$ años } & \multicolumn{2}{c}{$\mathbf{1 4 - 1 8}$ años } \\
\hline & Último año & Último mes & Último año & Último mes \\
\hline Alcohol & $58,7 \%$ & $37,9 \%$ & 73,4 & 49,7 \\
$\begin{array}{l}\text { 3 o más } \\
\text { consumiciones }\end{array}$ & $41,8 \%$ & $25 \%$ & $55,1 \%$ & $33,9 \%$ \\
$\begin{array}{l}6 \text { o más } \\
\text { consumiciones }\end{array}$ & $24,5 \%$ & $12,8 \%$ & $33 \%$ & $17,2 \%$ \\
Emborracharse & $34,4 \%$ & $16,5 \%$ & $46 \%$ & $22,4 \%$ \\
\hline
\end{tabular}


habían emborrachado en los últimos 30 días se situaba en el $22,2 \%$.

Los resultados revelan también (Tabla 2) que existen diferencias significativas en el porcentaje de chicos y chicas que han consumido alcohol de forma intensiva tanto en el último año como en el último mes, con porcentajes mayores entre los varones, especialmente en el caso de la ingesta de 6 o más bebidas alcohólicas. Por otra parte, se ha podido comprobar que el CIA se incrementa significativamente con la edad, con porcentajes hasta 20 y 30 veces mayores en la franja de 16 a 18 años, en comparación con la de 1213 años. Con el objetivo de conocer entre qué grupos de edad se obtenían diferencias significativas se compararon los grupos dos a dos, señalando en la tabla (mediante los correspondientes superíndices) aquellos casos en los que las diferencias eran significativas. Los resultados obtenidos revelaron la existencia de diferencias significativas entre los tres grupos de edad, siendo sensiblemente mayores entre los grupos extremos (12-13 vs. 16-18) (Tabla 2).

Asimismo, los datos revelan también la existencia de diferencias estadísticamente significativas en función de la titularidad del centro educativo, encontrándose tasas mayores en los colegios públicos. Respecto al entorno, es posible señalar que los adolescentes que residen en el ámbito urbano presentan mayores tasas de CIA. Por último, se puede comprobar que las cifras de prevalencia se incrementan significativamente a medida que desciende el nivel de estudios de los padres, siendo en la ingesta de 3 o más bebidas alcohólicas durante el último año donde se registran las mayores diferencias. Por último, al igual que en el caso de la edad, se han realizado también comparaciones dos a dos, para saber entre qué grupos se obtenían realmente diferencias significativas. Como se puede ver en la Tabla 2, se obtienen diferencias significativas prácticamente entre todos los grupos, salvo entre los adolescentes cuyos padres tienen estudios primarios y estudios medios, cuando se analiza el consumo de 3 o más bebidas alcohólicas y la conducta de emborracharse.

\section{Riesgos asociados}

Tal y como muestran los resultados recogidos en la Tabla 3 , los adolescentes que han realizado un consumo intensivo de alcohol en el último año se implican significativamente más en todas las conductas de riesgo consideradas,

Tabla 2. Diferencias en el CIA según variables sociodemográficas.

\begin{tabular}{|c|c|c|c|c|c|c|c|c|c|c|}
\hline \multirow[b]{2}{*}{ Género } & \multicolumn{5}{|c|}{ Último año } & \multicolumn{5}{|c|}{ Último mes } \\
\hline & $\begin{array}{r}\text { Hombres } \\
(\%)\end{array}$ & Mujeres (\%) & & $\chi^{2}$ & $\phi$ & $\begin{array}{r}\text { Hombres } \\
(\%)\end{array}$ & Mujeres (\%) & & $\chi^{2}$ & $\phi$ \\
\hline 6 o más consumiciones & 29,2 & 19,2 & & $38,65^{\star \star}$ & ,12 & 16 & 9,2 & & $29,47^{\star \star}$ &, 10 \\
\hline Emborracharse & 36 & 32,5 & & $3,89^{*}$ &, 04 & 18,2 & 14,6 & & $6,63^{\star}$ &, 05 \\
\hline 3 o más consumiciones & $5,1^{2,3}$ & $32,5^{1,3}$ & $72,1^{1,2}$ & $926,62^{\star \star}$ & ,49 & $1,3^{2,3}$ & $14,4^{1,3}$ & $48,7^{1,2}$ & $642,43^{\star \star}$ & ,43 \\
\hline 6 o más consumiciones & $1,3^{2,3}$ & $15,5^{1,3}$ & $46,2^{1,2}$ & $577,95^{\star *}$ & ,41 & $0,8^{2,3}$ & $6,3^{1,3}$ & $25,4^{1,2}$ & 307,90 ** & ,31 \\
\hline Emborracharse & $2,9^{2,3}$ & $25,4^{1,3}$ & $61,6^{1,2}$ & $775,26^{\star \star}$ & ,46 & $0,5^{2,3}$ & $9,2^{1,3}$ & $32,4^{1,2}$ & $398,63^{\star \star}$ &, 35 \\
\hline Titularidad & $\begin{array}{r}\text { Público } \\
(\%)\end{array}$ & $\begin{array}{r}\text { Privado } \\
(\%)\end{array}$ & & $\chi^{2}$ & $\phi$ & $\begin{array}{r}\text { Público } \\
(\%)\end{array}$ & $\begin{array}{r}\text { Privado } \\
(\%)\end{array}$ & & $\chi^{2}$ & $\phi$ \\
\hline 6 o más consumiciones & 27,3 & 18,4 & & $26,26^{\star \star}$ & ,10 & 14,9 & 8,1 & & $25,27^{\star \star}$ & ,09 \\
\hline Emborracharse & 37,4 & 28 & & 23,90 ** & 09 & 17,7 & 13,7 & & $6,97^{\star}$ &, 05 \\
\hline Entorno & $\begin{array}{r}\text { Rural } \\
(\%)\end{array}$ & $\begin{array}{r}\text { Urbano } \\
(\%)\end{array}$ & & $\chi^{2}$ & $\phi$ & $\begin{array}{r}\text { Rural } \\
(\%)\end{array}$ & $\begin{array}{r}\text { Urbano } \\
(\%)\end{array}$ & & $\chi^{2}$ & $\phi$ \\
\hline 3 o más consumiciones & 38 & 44,5 & & $12,02^{\star \star}$ & 07 & 22,9 & 26,6 & & $4,79^{\star}$ &, 04 \\
\hline 6 o más consumiciones & 21,9 & 26,5 & & $7,97^{\star}$ & 05 & 11 & 14,2 & & $6,04^{\star}$ & ,05 \\
\hline Emborracharse & 30,7 & 37,2 & & $13,30 * \star$ & 07 & 13,9 & 18,4 & & $9,75^{\star}$ & ,06 \\
\hline Estudios padres & $\begin{array}{r}\text { Primarios }^{1} \\
(\%)^{\mathrm{a}}\end{array}$ & $\begin{array}{r}\text { Medios }^{2} \\
(\%)^{b}\end{array}$ & $\begin{array}{r}\text { Superiores }^{3} \\
(\%)^{c}\end{array}$ & $\chi^{2}$ & $C C$ & $\begin{array}{r}\text { Primarios }^{1} \\
(\%)\end{array}$ & $\begin{array}{r}\text { Medios }^{2} \\
(\%)\end{array}$ & $\begin{array}{r}\text { Superiores } \\
(\%)\end{array}$ & $\chi^{2}$ & $C C$ \\
\hline 3 o más consumiciones & $48,9^{3}$ & $46,2^{3}$ & $33,5^{1,2}$ & $52,01^{\star *}$ & 14 & $30,5^{3}$ & $28,5^{3}$ & $18,5^{1,2}$ & 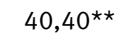 & ,12 \\
\hline 6 o más consumiciones & $30,6^{2,3}$ & $26,1^{1,3}$ & $19,5^{1,2}$ & $29,13^{\star \star}$ & ,10 & $18,1^{2,3}$ & $14^{1,3}$ & $8,6^{1,2}$ & $34,75^{\star \star}$ & ,11 \\
\hline
\end{tabular}

Nota. ${ }^{1,2,3}$ Grupos con los que se han encontrado diferencias significativas $(p<, 05)$. a: Ambos tienen estudios primarios o sin estudios; b: Al menos uno tiene estudios medios; c: Al menos uno tiene estudios universitarios. ${ }^{\star} p<, 05 .{ }^{\star \star} p<, 001$. 
especialmente en el caso de peleas, accidentes o lesiones y relaciones sexuales sin protección. Si tenemos en cuenta el valor de los coeficientes phi, cabe señalar además que son los adolescentes que consumen 6 o más bebidas alcohólicas los que presentan un mayor riesgo de sufrir todo este tipo de consecuencias. Asimismo, se ha podido corroborar la existencia de diferencias estadísticamente significativas entre consumidores intensivos vs. no intensivos en cuanto al consumo de otras sustancias, especialmente tabaco y cannabis (Tabla 4).

No menos interesante ha sido constatar que el porcentaje de adolescentes que podrían estar realizando un consumo de riesgo de alcohol, evaluado específicamente a través del AUDIT, se incrementa significativamente en- tre los consumidores intensivos. Nuevamente, los mayores porcentajes se obtienen entre quienes han ingeridos 6 o más bebidas alcohólicas en el último año $(81,3 \%)$, seguido de los que se emborracharon $(66 \%)$ y de los que consumieron 3 o más bebidas alcohólicas $(58,5 \%)$, situándose el porcentaje de positivos en el AUDIT para la muestra global en el $25,7 \%\left(\chi^{2}=1560,73 ; p<, 001\right)$.

\section{Posibles factores de pronóstico}

Tal y como se refleja en la Tabla 5, se han encontrado diferencias estadísticamente significativas en prácticamente todas las creencias exploradas, lo que pone de manifiesto que aquellos que hicieron un consumo intensivo en el último año en cualquiera de sus formas sobrevaloran los efec-

Tabla 3. Prácticas de riesgo (últimos 12 meses).

\begin{tabular}{|c|c|c|c|c|c|c|c|c|c|c|c|c|}
\hline & \multicolumn{2}{|c|}{$\begin{array}{c}3 \text { o más } \\
\text { consumiciones }\end{array}$} & \multirow{2}{*}{$\chi^{2}$} & \multirow{2}{*}{$\phi$} & \multicolumn{2}{|c|}{$\begin{array}{c}6 \text { o más } \\
\text { consumiciones }\end{array}$} & \multirow{2}{*}{$\chi^{2}$} & \multirow{2}{*}{$\phi$} & \multicolumn{2}{|c|}{ Emborracharse } & \multirow{2}{*}{$\chi^{2}$} & \multirow{2}{*}{$\phi$} \\
\hline & Sí (\%) & No (\%) & & & Sí (\%) & No (\%) & & & Sí (\%) & No (\%) & & \\
\hline $\begin{array}{l}\text { Accidentes o } \\
\text { lesiones }\end{array}$ & 16,7 & 1,6 & $221,87^{\star *}$ & ,28 & 22,8 & 3 & $291,40^{\star \star}$ &, 32 & 19,5 & 1,8 & $281,41^{\star *}$ & ,31 \\
\hline $\begin{array}{l}\text { Problemas con los } \\
\text { padres }\end{array}$ & 10,9 & 1,1 & $140,43^{* *}$ & ,22 & 13,7 & 2,4 & $138,78^{\star *}$ & ,22 & 12,2 & 1,5 & $151,95^{* *}$ & ,23 \\
\hline Peor rendimiento & 9 & 0,8 & $115,75^{\star \star}$ & ,20 & 11,9 & 1,7 & $135,96^{\star *}$ & ,22 & 10,5 & 0,9 & $147,71^{\star \star}$ & ,23 \\
\hline $\begin{array}{l}\text { Víctima de atracos/ } \\
\text { robos }\end{array}$ & 3,9 & 0,3 & $50,99 * *$ & ,13 & 5 & 0,8 & $52,91^{\star *}$ & ,14 & 4,4 & 0,4 & $57,04^{\star \star}$ & ,14 \\
\hline $\begin{array}{l}\text { Problemas con la } \\
\text { policía }\end{array}$ & 8,1 & 0,8 & $103,61^{\star *}$ & ,19 & 11,6 & 1,4 & $147,15^{\star *}$ &, 23 & 9,8 & 0,7 & $145,09 * *$ &, 22 \\
\hline $\begin{array}{l}\text { Ir a urgencias/ser } \\
\text { hospitalizado }\end{array}$ & 5,6 & 0,8 & $57,56^{\star \star}$ & ,14 & 8,3 & 1,1 & $96,31^{\star *}$ & ,18 & 6,9 & 0,8 & $85,99^{\star \star}$ & ,17 \\
\hline $\begin{array}{l}\text { Relaciones sexuales } \\
\text { sin protección }\end{array}$ & 14,5 & 0,8 & $217,39^{\star \star}$ &, 27 & 21 & 1,9 & $319,85^{\star \star}$ &, 33 & 17,2 & 0,9 & $286,57^{\star \star}$ & ,31 \\
\hline $\begin{array}{l}\text { Relaciones sexuales } \\
\text { de las que te } \\
\text { arrepentiste }\end{array}$ & 13,1 & 0,6 & $198,52^{\star \star}$ & ,26 & 19,6 & 1,4 & $318,87^{\star *}$ & ,33 & 15,3 & 0,9 & $243,67^{\star *}$ & ,29 \\
\hline $\begin{array}{l}\text { Viajar con un } \\
\text { conductor bajo los } \\
\text { efectos del alcohol }\end{array}$ & 43,4 & 18,9 & $206,87^{\star \star}$ & ,27 & 51,9 & 21,7 & $237,65^{\star \star}$ & ,29 & 46,8 & 19,7 & $233,90^{\star *}$ & ,28 \\
\hline $\begin{array}{l}\text { Conducir bajo los } \\
\text { efectos del alcohol }\end{array}$ & 7,5 & 0,4 & $107,87^{\star *}$ & ,19 & 11,3 & 0,9 & $173,28^{\star *}$ &, 25 & 9,1 & 0,3 & 157,10 & ,24 \\
\hline
\end{tabular}

Nota. ${ }^{\star} p<, 05 .{ }^{* \star} p<, 001$

Tabla 4. Consumo de otras sustancias (últimos 12 meses).

\begin{tabular}{|c|c|c|c|c|c|c|c|c|c|c|c|c|}
\hline & \multicolumn{2}{|c|}{$\begin{array}{c}3 \text { o más } \\
\text { consumiciones }\end{array}$} & \multirow{2}{*}{$\chi^{2}$} & \multirow{2}{*}{$\phi$} & \multicolumn{2}{|c|}{$\begin{array}{c}6 \text { o más } \\
\text { consumiciones }\end{array}$} & \multirow{2}{*}{$\chi^{2}$} & \multirow{2}{*}{$\phi$} & \multicolumn{2}{|c|}{ Emborracharse } & \multirow{2}{*}{$\chi^{2}$} & \multirow{2}{*}{$\phi$} \\
\hline & Sí (\%) & No (\%) & & & Sí (\%) & No (\%) & & & Sí (\%) & No (\%) & & \\
\hline Tabaco & 62,5 & 7,5 & $1017,20^{\star \star}$ &, 59 & 72,2 & 16,5 & $792,97^{\star *}$ &, 52 & 68,6 & 10,1 & $1065,02^{\star \star}$ & ,61 \\
\hline Marihuana o hachís & 41,3 & 2,9 & 681,91 ** & ,48 & 53,7 & 7,7 & $739,71^{\star *}$ &, 50 & 47,8 & 3,6 & $838,36^{\star *}$ &, 54 \\
\hline Cocaína & 3,9 & 0,1 & $59,65^{\star \star}$ & , 15 & 6,2 & 0,3 & $104,43^{* *}$ & ,19 & 4,7 & 0,1 & $81,27^{\star \star}$ & ,17 \\
\hline $\begin{array}{l}\text { Éxtasis, anfetas } \\
\text { o alucinógenos }\end{array}$ & 5,7 & 0,1 & $91,44^{\star *}$ & ,18 & 8,8 & 0,4 & $159,76^{\star *}$ &, 24 & 6,5 & 0,2 & $113,14^{\star \star}$ & ,20 \\
\hline
\end{tabular}

Nota. ${ }^{\star} p<, 05 .{ }^{\star \star} p<, 001$. 
tos positivos del alcohol (en especial, "divertirse mucho", "sentirse feliz" y "sentirse sociable y extrovertido"), a la vez que infravaloran los efectos negativos (sobre todo "tener problemas con la policía”, "no poder parar de beber" o "perjudicar su salud"). Con respecto a la hora de llegada a casa, los análisis realizados indican que cuanto más tarde vuelve a casa un adolescente cuando sale, mayor es la tasa de consumo intensivo de alcohol (Tabla 6). De igual modo los porcentajes de CIA se incrementan a medida que aumenta la cantidad de dinero disponible.

En cuanto al consumo en el entorno familiar, los resultados recogidos en la Tabla 7 ponen de manifiesto que cuando los padres consumen alcohol de manera regular se obtienen también mayores tasas de CIA entre los ado- lescentes. No obstante, las mayores diferencias se observan cuando los hermanos consumen alcohol. Por último, tal y como se detalla en la Tabla 8, se ha encontrado un porcentaje significativo mayor de adolescentes con CIA cuando los iguales también consumen alcohol, se emborrachan, fuman tabaco o cuando consumen otras drogas.

\section{Discusión}

A pesar de que los resultados de la última Encuesta sobre el Uso de Drogas en Estudiantes de Enseñanzas Secundarias (ESTUDES 2014-2015) (Plan Nacional sobre Drogas, 2016) revelan un considerable descenso del consumo de alcohol entre la población escolar, las cifras de prevalencia siguen

Tabla 5. Creencias y expectativas.

\begin{tabular}{|c|c|c|c|c|c|c|c|c|c|c|c|c|}
\hline & \multicolumn{2}{|c|}{$\begin{array}{c}30 \text { más } \\
\text { consumiciones }\end{array}$} & \multirow[t]{2}{*}{$t$} & \multirow{2}{*}{$\eta^{2}$} & \multicolumn{2}{|c|}{$\begin{array}{c}60 \text { más } \\
\text { consumiciones }\end{array}$} & \multirow[t]{2}{*}{$t$} & \multirow{2}{*}{$\eta^{2}$} & \multicolumn{2}{|c|}{ Emborracharse } & \multirow{2}{*}{$t$} & \multirow{2}{*}{$\eta^{2}$} \\
\hline & Sí (M) & No (M) & & & Sí (M) & No (M) & & & Sí (M) & No (M) & & \\
\hline Sentirme relajado & 2,13 & 1,48 & $-14,33^{\star *}$ & ,26 & 2,18 & 1,61 & $-11,08^{\star \star}$ & ,20 & 2,14 & 1,55 & $-12,49 * *$ & ,23 \\
\hline Problemas policía & 1,33 & 2,22 & $17,30^{\star \star}$ & ,30 & 1,30 & 2,02 & $12,65^{\star *}$ &, 22 & 1,31 & 2,13 & $15,49^{\star \star}$ & ,27 \\
\hline Perjudicar mi salud & 2,52 & 3,07 & $11,14^{\star \star}$ & ,20 & 2,50 & 2,95 & $7,93^{\star \star}$ & ,15 & 2,53 & 3 & $9,17^{\star \star}$ & ,17 \\
\hline Sentirme feliz & 2,64 & 1,64 & $-22,47^{\star \star}$ &, 38 & 2,75 & 1,83 & $-18,77^{\star \star}$ &, 30 & 2,72 & 1,72 & $-22,31^{\star \star}$ &, 37 \\
\hline $\begin{array}{l}\text { Olvidar mis } \\
\text { problemas }\end{array}$ & 2,51 & 1,95 & $-11,21^{\star *}$ &, 21 & 2,63 & 2,03 & $-10,92^{\star \star}$ & ,19 & 2,59 & 1,97 & $-12,20^{\star \star}$ & ,22 \\
\hline $\begin{array}{l}\text { No poder parar } \\
\text { de beber }\end{array}$ & 1,31 & 2,12 & $16,31^{\star *}$ &, 28 & 1,41 & 1,90 & $8,78^{\star \star}$ & , 15 & 1,32 & 2,03 & $13,80^{\star \star}$ & ,24 \\
\hline Tener resaca & 2,62 & 2,80 & $3,61^{\star \star}$ & ,07 & 2,69 & 2,73 & 0,73 &, 01 & 2,69 & 2,74 & 1,02 &, 02 \\
\hline $\begin{array}{l}\text { Sentirme sociable } \\
\text { y extrovertido }\end{array}$ & 2,74 & 1,90 & $-18,01^{\star \star}$ & ,31 & 2,81 & 2,07 & $-14,26^{\star \star}$ &, 24 & 2,80 & 1,96 & $-17,70^{\star \star}$ &, 30 \\
\hline $\begin{array}{l}\text { Hacer algo de lo } \\
\text { que me arrepienta }\end{array}$ & 2,25 & 2,73 & $9,59^{\star \star}$ & ,18 & 2,35 & 2,59 & $4,33^{\star *}$ & ,08 & 2,33 & 2,64 & $6,25^{\star *}$ & ,11 \\
\hline Divertirme mucho & 2,93 & 1,83 & $-25,79^{\star \star}$ & ,42 & 3,08 & 2,02 & $-23,34^{\star \star}$ &, 36 & 3,03 & 1,90 & $-26,63^{* \star}$ & ,42 \\
\hline Encontrarme mal & 2,18 & 2,86 & 13,90 ** &, 25 & 2,10 & 2,73 & $11,67^{\star \star}$ &, 20 & 2,16 & 2,79 & $12,81^{\star \star}$ & ,22 \\
\hline
\end{tabular}

Nota. ${ }^{\star} p<, 05 .{ }^{* \star} p<, 001$

Tabla 6. Hora de llegada y dinero disponible.

\begin{tabular}{|c|c|c|c|c|c|c|c|c|c|c|c|c|c|}
\hline & & \multicolumn{2}{|c|}{$\begin{array}{c}30 \text { más } \\
\text { consumiciones }\end{array}$} & \multirow{2}{*}{$\chi^{2}$} & \multirow{2}{*}{$C C$} & \multicolumn{2}{|c|}{$\begin{array}{c}6 \text { o más } \\
\text { consumiciones }\end{array}$} & \multirow{2}{*}{$\chi^{2}$} & \multirow{2}{*}{$C C$} & \multicolumn{2}{|c|}{ Emborracharse } & \multirow{2}{*}{$\chi^{2}$} & \multirow{2}{*}{$C C$} \\
\hline & & Sí (\%) & No (\%) & & & Sí (\%) & No (\%) & & & Sí (\%) & No (\%) & & \\
\hline \multirow{5}{*}{$\begin{array}{l}\text { Hora de } \\
\text { llegada }\end{array}$} & Antes de las $12 \mathrm{~h}$ & 5,4 & 94,6 & \multirow{5}{*}{$865,30^{* *}$} & \multirow{5}{*}{, 52} & 2,1 & 97,9 & \multirow{5}{*}{$672,94^{\star *}$} & \multirow{5}{*}{,47 } & 3,8 & 96,2 & \multirow{5}{*}{$775,42^{\star \star}$} & \multirow{5}{*}{, 50} \\
\hline & Entre las $12-2 \mathrm{~h}$ & 26,2 & 73,8 & & & 8,9 & 91,1 & & & 17 & 83 & & \\
\hline & Entre las 2-4h & 58,3 & 41,7 & & & 26,8 & 73,2 & & & 45,1 & 54,9 & & \\
\hline & Entre las 4-6h & 84 & 16 & & & 57,7 & 42,3 & & & 73,5 & 26,5 & & \\
\hline & Más tarde de las $6 \mathrm{~h}$ & 92,1 & 7,9 & & & 77 & 23 & & & 88,1 & 11,9 & & \\
\hline \multirow{5}{*}{ Dinero } & $0 €$ & 22,4 & 77,6 & \multirow{5}{*}{$294,99 * \star$} & \multirow{5}{*}{, 33} & 11,8 & 88,2 & \multirow{5}{*}{$268,82^{\star \star}$} & \multirow{5}{*}{, 32} & 19,7 & 80,3 & \multirow{5}{*}{$195,22^{\star *}$} & \multirow{5}{*}{,28 } \\
\hline & Hasta $10 €$ & 31,1 & 68,9 & & & 14,6 & 85,4 & & & 26,1 & 73,9 & & \\
\hline & Entre $11 €$ y $20 €$ & 52 & 48 & & & 27,8 & 72,2 & & & 42,9 & 57,1 & & \\
\hline & Entre $21 €$ y $30 €$ & 70 & 30 & & & 46,4 & 53,6 & & & 54 & 46 & & \\
\hline & Más de $30 €$ & 82,3 & 17,7 & & & 62,1 & 37,9 & & & 70 & 30 & & \\
\hline
\end{tabular}

Nota. ${ }^{\star} p<, 05 .{ }^{* \star} p<, 001$ 
Tabla 7. Consumo de alcohol en el entorno familiar.

\begin{tabular}{|c|c|c|c|c|c|c|c|c|c|c|c|c|c|}
\hline \multirow{2}{*}{\multicolumn{2}{|c|}{ Consumo alcohol }} & \multicolumn{2}{|c|}{$\begin{array}{c}3 \text { o más } \\
\text { consumiciones }\end{array}$} & \multirow[t]{2}{*}{$\chi^{2}$} & \multirow[t]{2}{*}{$\phi$} & \multicolumn{2}{|c|}{$\begin{array}{c}60 \text { más } \\
\text { consumiciones }\end{array}$} & \multirow[t]{2}{*}{$\chi^{2}$} & \multirow[t]{2}{*}{$\Phi$} & \multicolumn{2}{|c|}{ Emborracharse } & \multirow[t]{2}{*}{$\chi^{2}$} & \multirow[t]{2}{*}{$\phi$} \\
\hline & & Sí (\%) & No (\%) & & & Sí (\%) & No (\%) & & & Sí (\%) & No (\%) & & \\
\hline Madre & Regularmente & 44,8 & 55,2 & $4,58^{\star}$ &, 04 & 27,2 & 72,8 & $5,03^{*}$ &, 04 & 37 & 63 & 3,70 & ,04 \\
\hline Padre & Casi nunca & 36 & 64 & $28,86^{\star \star}$ & ,10 & 20,2 & 79,8 & $20,80^{\star \star}$ & ,09 & 30,1 & 69,9 & $16,67^{\star *}$ & ,08 \\
\hline \multirow{2}{*}{ Hermanos } & Casi nunca & 32,6 & 67,4 & \multirow{2}{*}{$168,48^{\star \star}$} & \multirow{2}{*}{, 26} & 18 & 82 & \multirow{2}{*}{112,90 ** } & \multirow{2}{*}{, 21} & 26,4 & 73,6 & \multirow{2}{*}{137,91 ** } & \multirow{2}{*}{,24 } \\
\hline & Regularmente & 59,3 & 40,7 & & & 37,2 & 62,8 & & & 49,8 & 50,2 & & \\
\hline
\end{tabular}

Nota. ${ }^{\star} p<, 05 .{ }^{\star \star} p<<, 001$

Tabla 8. Consumo de alcohol y otras sustancias entre los iguales.

\begin{tabular}{|c|c|c|c|c|c|c|c|c|c|c|c|c|c|}
\hline & & \multicolumn{2}{|c|}{$\begin{array}{c}30 \text { más } \\
\text { consumiciones }\end{array}$} & \multirow{2}{*}{$\chi^{2}$} & \multirow[t]{2}{*}{$C C$} & \multicolumn{2}{|c|}{$\begin{array}{c}6 \text { o más } \\
\text { consumiciones }\end{array}$} & \multirow{2}{*}{$\chi^{2}$} & \multirow[t]{2}{*}{$C C$} & \multicolumn{2}{|c|}{ Emborracharse } & \multirow{2}{*}{$\chi^{2}$} & \multirow[t]{2}{*}{$C C$} \\
\hline & & Sí (\%) & No (\%) & & & Sí (\%) & No (\%) & & & Sí (\%) & No (\%) & & \\
\hline \multirow{4}{*}{$\begin{array}{l}\text { Beben } \\
\text { alcohol }\end{array}$} & Ninguno/a & 1,4 & 98,6 & \multirow{4}{*}{$1231,17^{\star \star *}$} & \multirow{4}{*}{, 54} & 0,3 & 99,7 & \multirow{4}{*}{$776,57^{\star \star}$} & \multirow{4}{*}{,46 } & 0,5 & 99,5 & \multirow{4}{*}{$1033,93^{\star \star}$} & \multirow{4}{*}{, 51} \\
\hline & Pocos/as & 11,1 & 88,9 & & & 5 & 95 & & & 9,5 & 90,5 & & \\
\hline & Algunos/as & 35,9 & 64,1 & & & 14,7 & 85,3 & & & 23,7 & 76,3 & & \\
\hline & Todos/as & 90,4 & 9,6 & & & 66,9 & 33,1 & & & 79,8 & 20,2 & & \\
\hline \multirow{4}{*}{$\begin{array}{l}\text { Se } \\
\text { emborrachan }\end{array}$} & Ninguno/a & 3,9 & 96,1 & \multirow{4}{*}{$1009,38^{\star \star}$} & \multirow{4}{*}{, 51} & 1 & 99 & \multirow{4}{*}{$719,15^{\star \star}$} & \multirow{4}{*}{,44 } & 1 & 99 & \multirow{4}{*}{$1042,63^{\star \star}$} & \multirow{4}{*}{, 51} \\
\hline & Pocos/as & 31,7 & 68,3 & & & 13,1 & 86,9 & & & 20,7 & 79,3 & & \\
\hline & Algunos/as & 60,2 & 39,8 & & & 32,1 & 67,9 & & & 46,8 & 53,2 & & \\
\hline & La mayoría & 74,1 & 25,9 & & & 51,8 & 48,2 & & & 71 & 29 & & \\
\hline \multirow{5}{*}{$\begin{array}{l}\text { Consumen } \\
\text { tabaco }\end{array}$} & Ninguno/a & 7,4 & 92,6 & \multirow{5}{*}{$749,53^{\star \star}$} & \multirow{5}{*}{,45 } & 2,2 & 97,8 & \multirow{5}{*}{$545,69^{\star \star}$} & \multirow{5}{*}{,40 } & 4,5 & 95,5 & \multirow{5}{*}{$715,76^{\star *}$} & \multirow{5}{*}{,44 } \\
\hline & Pocos/as & 37,6 & 62,4 & & & 16,6 & 83,4 & & & 27 & 73 & & \\
\hline & Algunos/as & 59,8 & 40,2 & & & 36,9 & 63,1 & & & 50,1 & 49,9 & & \\
\hline & La mayoría & 72,5 & 27,5 & & & 50,4 & 49,6 & & & 66,6 & 33,4 & & \\
\hline & Todos/as & 86,4 & 13,6 & & & 67,2 & 32,8 & & & 84,4 & 15,6 & & \\
\hline \multirow{5}{*}{$\begin{array}{l}\text { Consumen } \\
\text { otras drogas }\end{array}$} & Ninguno/a & 22,8 & 77,2 & & & 10,4 & 89,6 & & & 15,4 & 84,6 & & \\
\hline & Pocos/as & 58,1 & 41,9 & 58105 ** & 41 & 33 & 67 & $x 4,0^{x}$ & 38 & 48,6 & 51,4 & 06 6** & \\
\hline & Algunos/as & 71,2 & 28,8 & (0) & 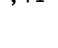 & 49,9 & 50,1 & 年 & & 64,7 & 35,3 & & \\
\hline & La mayoría & 81 & 19 & & & 58,2 & 41,8 & & & 81,1 & 18,9 & & \\
\hline & Todos/as & 89,5 & 10,5 & & & 82,1 & 17,9 & & & 91,9 & 8,1 & & \\
\hline
\end{tabular}

Nota. ${ }^{\star} p<, 05 .{ }^{* \star} p<, 001$.

siendo elevadas, especialmente en lo que se refiere al consumo intensivo. Habida cuenta de que la edad de inicio en el consumo de alcohol y otras sustancias es cada vez más precoz, en el presente trabajo se optó por ampliar el marco muestral a los 12-18 años, motivados por la extensa literatura que advierte de las graves consecuencias de este patrón de consumo a edades muy tempranas (Ellickson, Tucker, y Klein, 2003; Motos et al., 2015; Stueve y O`Donnell, 2005). Los resultados obtenidos ponen de manifiesto que el CIA es una práctica habitual y globalizada (entre el $24,5 \%$ y el $41,8 \%$ de los adolescentes gallegos). A pesar de que los porcentajes encontrados en la franja de edad más temprana son reducidos (1,3\% para el consumo de 6 o más bebidas alcohólicas en el último año, 2,9\% para el hecho de emborracharse y $5,1 \%$ para el consumo de 3 o más bebidas alcohólicas), llevados a cifras poblacionales supone que entre 500 y 2.000 niños de entre 12 y 13 años de la comunidad gallega reconocen haber realizado un consumo intensivo de alcohol en el transcurso del último año.

Además de estimar la prevalencia del CIA con el presente estudio empírico se ha intentado obtener nuevas evidencias de la gravedad de esta práctica a distintos niveles. De acuerdo con los numerosos trabajos hallados en la literatura que relacionan el CIA con numerosas consecuencias 
negativas y prácticas de riesgo (Miller et al., 2007; Wechsler et al., 1994) se ha observado que los adolescentes que presentan este patrón de consumo se involucran significativamente más en todas las conductas de riesgo consideradas. Asimismo se confirma la tendencia observada por otros autores (Chassin, Pitts, y Prost, 2002; Jones et al., 2001) en la que los adolescentes que realizan un consumo intensivo de alcohol presentan una mayor probabilidad de iniciarse en el consumo de otras sustancias, así como de desarrollar un consumo de riesgo (constatado a través del AUDIT) o incluso un posible trastorno o dependencia en la edad adulta (Norström y Pape, 2012; Viner y Taylor, 2007). Desde un punto de vista comparativo, de los tres patrones analizados los que realizan un consumo más intensivo (6 o más bebidas alcohólicas) son los que muestran una mayor probabilidad de involucrarse en las distintas prácticas de riesgo consideradas. No obstante, los que se emborrachan presentan niveles de policonsumo similares.

Desarrollar una respuesta eficaz en términos preventivos pasa por identificar algunas de las variables asociadas al CIA. En este sentido, se ha podido comprobar que los adolescentes que hacen un consumo intensivo presentan creencias y expectativas claramente positivas respecto a los efectos del alcohol, mucho más (comparativamente) que aquellos que hacen un consumo más moderado, en la línea de los planteamientos de Cortés et al. (2011) y McBride et al. (2014). Asimismo, el consumo tanto de alcohol como de otras sustancias por parte de los iguales ha mostrado ser una variable estrechamente relacionada con el CIA. Según Kandel y Andrews (1987), sería la imitación de la conducta de los iguales la forma dominante de influencia social, favoreciendo la selección de compañías que refuerzan este tipo de conductas. De igual, manera se ha podido constatar la influencia del consumo del entorno familiar, lo cual resulta coherente con la teoría del aprendizaje social, que destaca la importancia de la identificación del sujeto con el modelo (Espada et al., 2008). Otras dos variables que cuentan con una menor evidencia empírica en la literatura son la hora de llegada a casa y el dinero disponible. Si bien ambas han sido relacionadas en algunos estudios previos con el consumo de alcohol (Humensky, 2010; Plan Nacional sobre Drogas, 2014; Varela et al., 2013), en este trabajo se ha podido constatar que también se encuentran asociadas al CIA.

Desde un punto de vista sociodemográfico, a pesar de que este patrón podría decirse que constituye a día de hoy un fenómeno globalizado, es posible identificar un perfil en el que la prevalencia del CIA es mayor. En concreto, los porcentajes encontrados son significativamente mayores entre los chicos de entre 16 y 18 años, en entornos urbanos, en centros públicos y entre aquellos cuyos padres tienen un bajo nivel de estudios.

$\mathrm{Si}$ intentamos integrar toda la información referida a las variables consideradas como posibles "antecedentes", además del perfil sociodemográfico mencionado, cabría señalar la existencia de un patrón asociado al CIA, definido fundamentalmente por sus creencias y expectativas (jóvenes que tienden a atribuir en sobremanera efectos positivos al consumo intensivo de alcohol), un elevado consumo entre sus iguales (no sólo de alcohol, sino también de tabaco y otras sustancias), una hora de llegada a casa más tardía (especialmente a partir de las cuatro de la madrugada), una mayor cantidad de dinero disponible para salir (especialmente más de $30 €$ ) y un mayor consumo en el entorno familiar, sobre todo por parte de sus hermanos/as. La información disponible no permite establecer con rigor diferencias significativas entre los tres grupos de CIA considerados, si bien el conjunto de variables analizadas parece tener mayor capacidad para explicar o predecir el patrón de consumo más "moderado" (3 o más consumiciones), que para explicar el patrón más "severo" (6 o más consumiciones).

Por último, en cuanto a las posibles limitaciones de este trabajo cabe señalar, en primer lugar, la falta de consenso a la hora de hacer operativo la variable objeto de estudio, esto es, el consumo intensivo de alcohol. La falta de definición de una Unidad de Bebida Estándar (UBE) o la no especificación del período de tiempo que se considera "una única ocasión" convierte en una tarea verdaderamente difícil obtener una medida del CIA precisa y comparable entre unos países y otros. Para intentar atenuar este tipo de dificultades, en este trabajo se ha optado por utilizar 3 indicadores complementarios, dos de ellos cuantitativos ("haber consumido 3 o más bebidas alcohólicas en una misma ocasión" y "haber consumido 6 o más bebidas alcohólicas en una misma ocasión”) y otro más de tipo cualitativo o subjetivo ("haberse emborrachado"). Ello además ha proporcionado algunas "pistas" respecto a cómo se relaciona la percepción subjetiva del individuo con la cantidad objetiva de consumo. Un resultado que se repite en cada una de las cuestiones exploradas en este trabajo es que las cifras asociadas al hecho de emborracharse siempre se sitúan en el término medio entre el consumo de 3 o más bebidas alcohólicas y el de 6 o más. Cabría preguntarse, por tanto, si sería más conveniente operativizar el CIA como la ingesta de 4 o 5 consumiciones alcohólicas en una misma ocasión habida cuenta de que ello parece coincidir mejor con la percepción subjetiva de haberse emborrachado. Otra alternativa, quizás más apropiada para operativizar de forma rigurosa el CIA podría ser el identificar un conjunto de indicadores que incorpore tanto el dato objetivo del número de consumiciones como la percepción del propio individuo, intentando de algún modo desarrollar (y validar empíricamente) una escala breve de consumo intensivo de alcohol. Desde el punto de vista muestral, es importante hacer notar que si bien se ha trabajado con una muestra de 3.419 adolescentes que, en cierta medida, puede considerarse representativa de la comunidad autónoma de Galicia, cabe cuestionarse la capacidad de extrapolación a otras comunidades. Finalmente es importante advertir 
que estamos ante un trabajo de carácter exploratorio que no permite establecer relaciones de causalidad. A pesar de que conceptualmente es posible anticipar qué variables podrían estar actuando como predictores o como consecuentes del consumo intensivo, sólo un diseño longitudinal podría confirmar relaciones causales.

\section{Reconocimientos}

Los autores quieren agradecer la financiación recibida a través de la Delegación del Gobierno para el Plan Nacional sobre Drogas (Ref. 2013/046) para la realización de este estudio.

\section{Conflicto de intereses}

Los autores declaran no tener ningún conflicto de intereses.

\section{Referencias}

Adams, T. B., Evans, D. R., Shreffler, R. M. y Beam, K. J. (2006). Development and evaluation of theory-based alcohol education programs. Journal of Alcohol E Drug Education, 50, 21-30.

Anderson, P. (2007). Binge drinking and Europe. Hamm, Germany: German Centre for Addiction Issues (DHS). Recuperado de http://www.drugsandalcohol. ie/6353/1/3836-4088.pdf.

Anderson, P., Cremona, A., Paton, A., Turner, C. y Wallace, P. (1993). The risk of alcohol. Addiction, 88, 1493-1508.

Cadaveira, F. (2009). Alcohol y cerebro adolescente. Adicciones, 21, 9-14.

Cadaveira, F. (mayo, 2010). Consecuencias neurocognitivas: estudio de seguimiento en jóvenes españoles que realizan binge drinking. Trabajo presentando en el Seminario Consumo Intensivo de Alcohol en Jóvenes: conocimiento, alternativas y viabilidad, Valencia.

Calafat, A. (2007). El abuso de alcohol de los jóvenes en España. Adicciones, 19, 217-224.

Chambers, R.A., Taylor, J.R. y Potenza, M.N. (2003). Developmental neurociruitry of motivation in adolescence: A critical period of addiction vulnerability. American Journal of Psychiatry, 160, 1041-1052. doi:10.1176/appi. ajp.160.6.1041.

Chassin, L., Pitts, S. C. y Prost, J. (2002). Binge drinking trajectories from adolescence to emerging adulthood in a high-risk sample: Predictors and substance abuse outcomes. Journal of Consulting and Clinical Psychology, 70, 67-78. doi:10.1037//0022-006X.70.1.67.

Coleman, L. y Cater, S. (2005). Underage «binge» drinking: A qualitative study into motivations and outcomes. Drugs: Education, Prevention and Policy, 12, 125-136. doi:1 0.1080/09687630512331323521.
Consejo de la Unión Europea. (2013). Estrategia en materia de lucha contra las drogas de la Unión Europea 2013-2020. Luxemburgo, Luxemburgo: Publications Office of the European Union.

Cortés, M. T., Espejo, B. y Giménez, J. A. (2007). Características que definen el fenómeno del botellón en universitarios y adolescentes. Adicciones, 19, 357-372.

DeCamp, W., Gealt, R., Martin, S., O'Connell, D. y Visher, C. (2015). Binge drinking and other risk behaviors among college students. Newark, DE: University of Delaware.

Donovan, J. E. (2009). Estimated blood alcohol concentrations for child and adolescents drinking and their implications for screening instruments. Pediatrics, 123, e975-e981. doi:10.1542/peds.2008-0027.

Doumas, D. M., Turrisi, R. y Wright, D. A. (2006). Risk factors for heavy drinking and associated consequences in college freshmen: Athletic status and adult attachment. Sport Psychologist, 20, 419-434.

Durkin, K. F., Wolfe, T. W. y Clark, G. A. (2005). College students and binge drinking: An evaluation of social learning theory. Sociological Spectrum, 25, 255-272. doi:10.1080/027321790518681.

Ellickson, P., Tucker, J. y Klein, D. (2003). Ten-year prospective study of public health problems associated with early drinking. Pediatrics, 111, 949-955.

Espada, J. P., Pereira, J. R. y García-Fernández, J. M. (2008). Influencia de los modelos sociales en el consumo de alcohol de los adolescentes. Psicothema, 20, 531-537.

Fuller-Thomson, E., Sheridan, M. P., Sorichetti, C. y Mehta, R. (2013). Underage binge drinking adolescents: Sociodemographic profile and utilization of family doctors, 2013, 1-9. doi:10.5402/2013/728730.

García-Moreno, L. M., Expósito, J., Sanhueza, C. y Angulo, M. T. (2008). Actividad prefrontal y alcoholismo de fin de semana en jóvenes. Adicciones, 20, 271-279.

Guerri, C. (mayo, 2010). Neurotoxicidad y alteraciones cognitivas y conductuales. Trabajo presentando en el Seminario Consumo Intensivo de Alcohol en Jóvenes: conocimiento, alternativas y viabilidad, Valencia.

Guerri, C. y Pascual, M. (2010). Mechanisms involved in the neurotoxic, cognitive, and neurobehavioral effects of alcohol consumption during adolescence. Alcohol, 44, 15-26. doi:10.1016/j.alcohol.2009.10.003.

Hedden, S. L., Kennet, J., Lipari, R., Medley, G., Tice, P., Copello, E. A. P. y Kroutil, L. A. (2015). Behavioral health trends in the United States: Results from the 2014 national survey on drug use and health. Rockville, EE.UU: Substance Abuse and Mental Health Services Administration (SAMHSA). Recuperado de http://www.samhsa.gov/data/sites/default/files/NSDUH-FRR1-2014/ NSDUH-FRR1-2014.pdf.

Hibell, B., Guttormsson, U., Ahlström, S., Balakireva, O., Bjarnason, T., Kokkevi, A. y Kraus, L. (2012). The 2011 ESPAD Report: Substance Use Among Students in 36 
European Countries. Sweden: The Swedish Council for Information on Alcohol and Other Drugs (CAN). Recuperado de http://www.can.se/contentassets/ $8 \mathrm{~d} 8 \mathrm{cb}-$ 78bbd28493b9030c65c598e3301/the_2011_espad_report_full.pdf.

Huang, J. H., Jacobs, D. F. y Deverensky, J. L. (2010). Sexual risk-taking behaviors, gambling, and heavy drinking among U.S. college athletes. Archives of Sexual Behavior, 39, 706-713.

Humensky, J. L. (2010). Are adolescents with high socioeconomic status more likely to engage in alcohol and illicit drug use in early adulthood? Substance Abuse Treatment, Prevention, and Policy, 5, 19-29. doi:10.1186/1747597X-5-19.

Jander, A., Mercken, L., Crutzen, R. y De Vries, H. (2013). Determinants of binge drinking in a permissive environment: Focus group interviews with Dutch adolescents and parents. BMC Public Health, 13, 882-895. doi:10.1186/1471-2458-13-882.

Jones, S. E., Oeltmann, J., Wilson, T. W., Brener, N. D. y Hill, C. V. (2001). Binge drinking among undergraduate college students in the United States: Implications for other substance use. Journal of American College Health, 50, 33-38. doi:10.1080/ 07448480109595709.

Kandel, D. B. y Andrews, K. (1987). Processes of adolescent socialization by parents and peers. International Journal of Addictions, 22, 319-349.

López-Caneda, E., Mota, N., Crego, A., Velasquez, T., Corral, M., Rodríguez, S. y Cadaveira, F. (2014). Anomalías neurocognitivas asociadas al consumo intensivo de alcohol (binge drinking) en jóvenes y adolescentes: una revisión. Adicciones, 26, 334-359.

Marmot, M. G. (2001). Alcohol and coronary heart disease. International Journal of Epidemiology, 30, 724-729. doi:10.1093/ije/30.4.724.

McBride, N. M., Barret, B., Moore, K. A. y Schonfeld, L. (2014). The role of positive alcohol expectancies in underage drinking among college students. Journal of American College Health, 62, 370-379. doi:10.1080/07448481. 2014.907297.

Miller, J. W., Naimi, T. S., Brewer, R. D. y Jones, S. E. (2007). Binge drinking and associated health risk behaviors among high school students. Pediatrics, 119, 76-85. doi:10.1542/peds.2006-1517.

Mongan, D. y Long, J. (2015). Standard drink measures throughout Europe; people's understanding of standard drinks and their use in drinking guidelines, alcohol survey and labelling. Dublín, Irlanda: Reducing Alcohol Related Harm (RARHA). Recuperado de http://www.rarha.eu/Resources/ Deliverables/Lists/Deliverables/Attachments/14/WP5 Background paper Standard drink measures HRB.pdf.

Mota, N., Álvarez-Gil, R., Corral, M., Rodríguez, S., Parada, M., Crego, A., ... Cadaveira, F. (2010). Risky alcohol use and heavy episodic drinking among Spanish university students: A two-year follow-up. Gaceta Sanitaria, 24, 372377. doi:10.1016/ j.gaceta.2010.02.013.

Motos, P., Cortés, M. T., Giménez, J. A. y Cadaveira, F. (2015). Predictores del consumo semanal de alcohol y sus consecuencias asociadas en universitarios consumidores intensivos de alcohol. Adicciones, 27, 119-131.

National Institute on Alcohol Abuse and Alcoholism. (2004). NIAAA council approves definition of binge drinking. NIAAA Newsletter, (3), 3. Recuperado de http:/ / pubs.niaaa.nih.gov/publications/Newsletter/ winter2004/Newsletter_Number3.pdf.

Norström, T. y Pape, H. (2012). Associations between adolescent heavy drinking and problem drinking in early adulthood: Implications for prevention. Journal of Studies on Alcohol and Drugs, 73, 542-548. doi:10.15288/ jsad.2012.73.542.

Organización Mundial de la Salud. (2004). Global status report on alcohol 2004. Génova, Suiza: Department of Mental Health and Substance Abuse.

Parada, M., Corral, M., Caamaño-Isorna, F., Mota, N., Crego, A., Rodríguez Holguín, S. y Cadaveira, F. (2011). Definición del concepto de consumo intensivo de alcohol adolescente (binge drinking). Adicciones, 23, 53-63.

Peralta, R. L., Steele, J. L., Nofziger, S. y Rickles, M. (2010). The impact of gender on binge drinking behavior among U.S. college students attending a Midwestern university: An analysis of two gender measures. Feminist Criminology, 5, 355-379. doi:10.1177/ 1557085110386363.

Petit, G., Maurage, P., Kornreich, C., Verbanck, P. y Campanella, S. (2014). Binge drinking in adolescents: A review of neurophysiological and neuroimaging research. Alcohol and Alcoholism, 49, 198-206. doi:10.1093/alcalc/ agt172.

Pincock, S. (2003). Binge drinking on rise in UK and elsewhere. The Lancet, 362, 1126-1127.

Pirkle, E. C. y Richter, L. (2006). Personality, attitudinal and behavioral risk profiles of young female binge drinkers and smokers. Journal of Adolescent Health, 38, 44-54.

Plan Nacional sobre Drogas. (2009a). Estrategia Nacional sobre Drogas 2009-2016. Madrid, España: Ministerio de Sanidad y Consumo.

Plan Nacional sobre Drogas. (2009b). Plan de Acción sobre Drogas, España 2013-2016. Madrid, España: Ministerio de Sanidad, Servicios Sociales e Igualdad.

Plan Nacional sobre Drogas. (2011). Encuesta sobre el uso de drogas en enseñanzas secundarias (ESTUDES) 2010. Madrid, España: Ministerio de Sanidad, Política Social e Igualdad.

Plan Nacional sobre Drogas. (2014). Encuesta sobre el uso de drogas en enseñanzas secundarias en España (ESTUDES) 2012-2013. Madrid, España: Ministerio de Sanidad, Servicios Sociales e Igualdad.

Plan Nacional sobre Drogas. (2016). Encuesta sobre el uso de drogas en enseñanzas secundarias en España (ESTUDES) 
2014-2015. Madrid, España: Ministerio de Sanidad, Servicios Sociales e Igualdad.

Pons, J. y Berjano, E. (1999). El consumo abusivo de alcohol en la adolescencia: un modelo explicativo desde la psicología social. Madrid, España: Plan Nacional sobre Drogas.

Rial, A., Gómez, P., Araujo, M., Real, E., Cartelle, J., Picón, E., ... Isorna, M. (2015, septiembre). Psychometric properties of the self-administered AUDIT in Spanish adolescents. Comunicación presentada en First European Conference on Addictive Behaviours and Dependencies. Lisboa, Portugal.

Rodríguez-Martos, A. y Rosón, B. (2008). Definición y terminología. En Ministerio de Sanidad y Consumo (Ed.), Prevención de los problemas derivados del alcohol: 1a Conferencia de prevención y promoción de la salud en la práctica clínica en España (pp. 39-48). Madrid, España: Ministerio de Sanidad y Consumo.

Stickley, A., Koyanagi, A., Koposov, R., McKee, M., Roberts, B., Murphy, A. y Ruchkin, V. (2013). Binge drinking among adolescents in Russia: Prevalence, risk and protective factors. Addictive Behaviors, 38, 1988-1995. doi:10.1016/j.addbeh.2012.12.009.

Stueve, A. y O’Donnell, L. N. (2005). Early alcohol initiation and subsequent sexual and alcohol risk behaviors among urban youths. American Journal of Public Health, 05, 887-893. doi:10.2105/AJPH.2003.026567.

Swahn, M. H., Simon, T., Hamming, B. J. y Guerrero, J. L. (2004). Alcohol-consumption behaviors and risk for physical fighting and injuries among adolescent drinkers. Addictive Behaviors, 29, 959-963. doi:10.1016/j. addbeh.2004.02.043.

Tapert, S.F. (abril, 2007). Consumo de alcohol en adolescentes: efectos sobre el cerebro. Trabajo presentando en el Seminario Internacional sobre alcohol y daño cerebral en menores, Madrid.

Tapert, S.F. y Brown, S.A. (1999). Neuropsychological correlates of adolescents substance abuse: Four-year outcomes. Journal of the International Neuropsychological Society, 5, 481-492. doi:10.1017/S1355617799566010.

Varela, J., Marsillas, S., Isorna, M. y Rial, A. (2013). El papel de las actitudes, las percepciones y el dinero disponible en el consumo de drogas en adolescentes. Salud y Drogas, 13, 67-78.

Viner, R. M. y Taylor, B. (2007). Adult outcomes of binge drinking in adolescence: Findings from a UK national birth cohort. Journal of Epidemiology $\mathcal{E}$ Community Health, 61, 902-907. doi:10.1136/jech.2005.038117.

Wechsler, H., Davenport, A., G., Dowdall, G., Moeykens, B. y Castillo, S. (1994). Health and behavioral consequences. Journal of the American Medical Association, 272, 16721677.

Windle, M. (2003). Alcohol use among adolescents and young adults. Alcohol Research and Health, 27, 79-85.
Windle, M., Mun, E. Y. y Windle, R. C. (2005). Adolescent to young adulthood heavy drinking trajectories and their prospective predictors. Journal of Studies on Alcohol, 66, 313-322. doi:10.15288/jsa.2005.66.313.

Xunta de Galicia. (2010). Plan de Trastornos Adictivos de Galicia 2011-2016. Santiago de Compostela, España: Consellería de Sanidade, Xunta de Galicia. Recuperado de http://xuventude.xunta.es/uploads/docs/ Observatorio/Plan_de_trastornos_adictivos_de_Galicia_2011-2016.pdf.

Zielger, D.W., Wang, C.C., Yoast, R.A., Dickinson, B.O., McCaffree, M.A., Robinowitz, C.B. y Sterling, M.L. (2005). The neurocognitive effects of alcohol on adolescents and college students. Preventive Medicine, 40, 23-32. doi:10.1016/j.ypmed.2004.04.044. 